\title{
Numerical Bubble Dynamics
}

\author{
Anton Smolianski ${ }^{a}$, Heikki Haario ${ }^{b}$ and Pasi Luukka ${ }^{b}$ \\ ${ }^{a}$ Institute of Mathematics \\ Zurich University \\ CH-8057 Zurich, Switzerland \\ email:anton.smolianski@math.unizh.ch \\ ${ }^{b}$ Laboratory of Applied Mathematics \\ Lappeenranta University of Technology \\ P.O. Box 20, FIN-53851 LPR, Finland \\ email:heikki.haario@lut.fi,pasi.luukka@lut.fi
}

\begin{abstract}
A computational study of the dynamics of a gas bubble rising in a viscous liquid is presented. The proposed numerical method allows to simulate a wide range of flow regimes, accurately capturing the shape of the deforming interface of the bubble and the surface tension effect, while maintaining a good mass conservation. With the present numerical method, the high-Reynolds number wobbling bubble regime exhibiting unsymmetric vortex path in the wake has been successfully simulated. The computed time-evolution of bubble's interface area, position and rise velocity shows a good agreement with the available experimental data. Some results on bubble coalescence phenomena are demonstrated. Our studies reveal that plausible results can be obtained with two-dimensional numerical simulations, when a single buoyant bubble or a coalescence of a pair of bubbles is considered.
\end{abstract}

\section{Introduction}

The rise of a gas bubble in a viscous liquid is a very complicated, non-linear and nonstationary hydrodynamical process. It is usually accompanied by a significant deformation of the bubble, indicating a complex interplay between fluid convection, viscosity and surface tension. The diverse shapes of the bubble resulting from this deformation cause a large variety of flow patterns around the bubble, and vice versa. A number of experimental studies have addressed this problem. Early studies include the rise of a bubble in an inviscid and a viscous liquid, see (Hartunian \& Sears 1957), (Walters \& Davidson 1962) (Walters \& Davidson 1963), (Wegener \& Parlange 1973) and (Bhaga \& Weber 1981). Approximate theoretical solutions have been obtained for either low (Taylor \& Acrivos 1964) or high (Moore 1959) Reynolds numbers under the assumption that the bubble remains nearly spherical.

We employ the level-set (see (Sethian 1999)) method that permits to compute topological changes of the interface (like mergers or breakups). We use the finite element method that relies on a global variational formulation and, thus, naturally incorporates the coefficient jumps and the singular interface-concentrated force. The combination of finite elements and the level-set technique allows us to localize the interface precisely, without an introduction of any artificial parameters like the interface thickness. As a whole, our computational method takes an advantage of combining the finite element spatial discretization, the operator-splitting temporal discretization and the level-set interface representation.

In (Tornberg 2000) a combination of the finite element and the level-set methods has been recently used to simulate a merger of two bubbles in a viscous liquid; however, the method is restricted to low Reynolds number flows only. 
Using the presented computational method we provide a systematic study of diverse shape regimes for a single buoyant bubble, recovering all main regimes in a full agreement with available experimental data (for detailed analysis see (Smolianski et al. )). Next, we present results on the bubble coalescence phenomena.

\section{Numerical method}

As a simulation tool we employ a general computational strategy proposed in (Smolianski 2001) (see also (Smolianski et al. )) that is capable of modeling any kind of two-fluid interfacial flows. The dynamics of a gas bubble in a liquid can, thus, be considered as a particular application of this computational approach.

We consider an unsteady laminar flow of two immiscible fluids. Both fluids are assumed to be viscous and Newtonian. Moreover, we suppose that the flow is isothermal, thus neglecting the viscosity and density variations due to changes of a temperature field. We assume also that the fluids are incompressible. Presuming, in addition, the fluids to be homogeneous, we may infer that the densities and viscosities are constant within each fluid. We utilize the sharp-interface (zero interfacial thickness) approach; the density and viscosity have, therefore, a jump discontinuity at the interface (see, e.g., (Batchelor 1967)). We assume that the interface has a surface tension. We also suppose that there is no mass transfer through the interface (i.e. the interface is impermeable), and there are no surfactants present in the fluids (hence, there is no species transport along the interface). The surface tension coefficient is, thus, assumed constant.

\section{Summary of the algorithm}

Our computational approach for numerical modelling of interfacial flows can be summarized as follows:

Step 0. Initialization of the level-set function and velocity.

For each $n$-th time-step, $n=1,2, \ldots$ :

1. Computation of interface normal and curvature.

2. Navier-Stokes convection step.

3. Viscous step.

4. Projection step.

5. Level-set convection step.

6. Reinitialization step.

7. Level-set correction step.

The steps 1.-7. are performed successively, and each of the steps 2.-5. may use its own local time-increment size. On each step the last computed velocity is exploited; the viscous and projection steps use the interface position found on the previous global timestep. It is also noteworthy that the steps 5.-7. can be computed in a fully parallel manner with the step 2 . The whole algorithm is very flexible; it permits, for instance, to compute unsteady interfacial Stokes flow just by omiting the Navier-Stokes convection step.

\section{Bubbles in different shape regimes}

Figure 1 shows the typical bubble shapes and velocity streamlines in the frame of reference of the bubble. Although all experimental results correspond to three-dimensional bubbles and our computations are only two-dimensional, a qualitative comparison is possible. The comparison enables us to conclude that our numerical bubble shapes are in a good agreement with the experimental predictions of (Bhaga \& Weber 1981) and (Clift et al. 1978). 


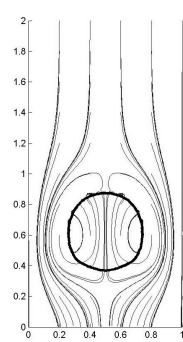

(a)

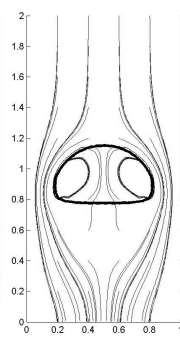

(b)

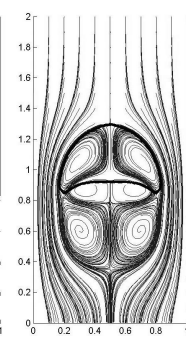

(c)

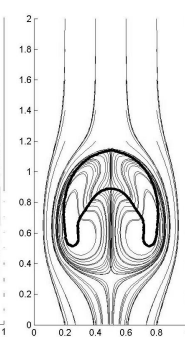

(d)

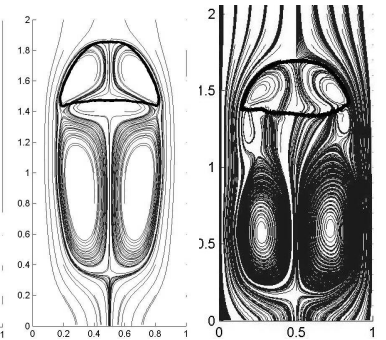

(e)

Figure 1. Different computed shapes of bubbles: (a) spherical with Re=1, Eo=0.6, (b) ellipsoidal with $R e=20$ Eo=1.2, (c) dimpled ellipsoidal cap with $R e=35, E o=125$, (d) skirted with $R e=55, E o=875$, (e) spherical cap with $R e=94, E o=115$ and $(f)$ wobbling with $\operatorname{Re}=1100, E o=3.0 ; \rho_{1} / \rho_{2}=10^{3} ; \mu_{1} / \mu_{2}=10^{2}$.

As it is seen from the figure, all basic shapes are successfully recovered with the parameter values lying exactly within the limits given in (Clift et al. 1978).

The interesting phenomena are observed in the case of a wobbling bubble. The wobbling typically appears with sufficiently high Reynolds numbers when the Eötvös number is, roughly, in the range between 1 and 100 (see (Clift et al. 1978)).

Since the typical range for the Reynolds number corresponding to the wobbling motion is approximately the same as for the spherical cap regime, the wobbling bubble (see Figure 2) retains a nearly spherical cap shape. However, at later stage of the motion, a remarkable flattening of the bubble top can be observed (Figure 2). The bubble bottom undergoes permanent deformations resulting from the unstable and unsymmetric evolution of the bubble wake. In particular, the unsymmetric pairs of secondary vortices are clearly observed in the wake as the consequence of asynchronous separation of the boundary layer from different sides of the bubble surface. This flow pattern bears some resemblance to the von Karman vortex path typically formed behind a rigid body in a highly convective flow. We are unaware of any other successful numerical simulations in the wobbling bubble regime.

\section{Results on coalescence of bubbles}

We consider the rectangular domain of the unit width with two initially circular bubbles inside; the radius of the upper bubble is equal to 0.25 and the radius of the lower one is 0.2 . Bubbles have a common axis of symmetry. We prescribe zero velocity field at the initial moment. The dynamics of the bubbles, to a large extent, depends on the initial distance between them and on the magnitude of the surface tension. If the surface tension is high enough, no merger happens, the bubbles develop nearly ellipsoidal shapes and rise separately (see, e.g., (Unverdi \& Tryggvason 1992)). Hence, in order to simulate a merger process, we take comparably small surface tension coefficient.

Figures 3-4 illustrate the process of bubble merger in different shape regimes.

During the rise of the bubble, two opposite signed vortices are created in the wake of the larger bubble. This produces a lower pressure region behind the large bubble and generates flow streaming into the symmetry line of the flow. As a result, the front portion of the small bubble becomes narrower and sharper. The head of the lower bubble almost catches up with the bottom of the upper one. In the next moment, the two bubbles merge 

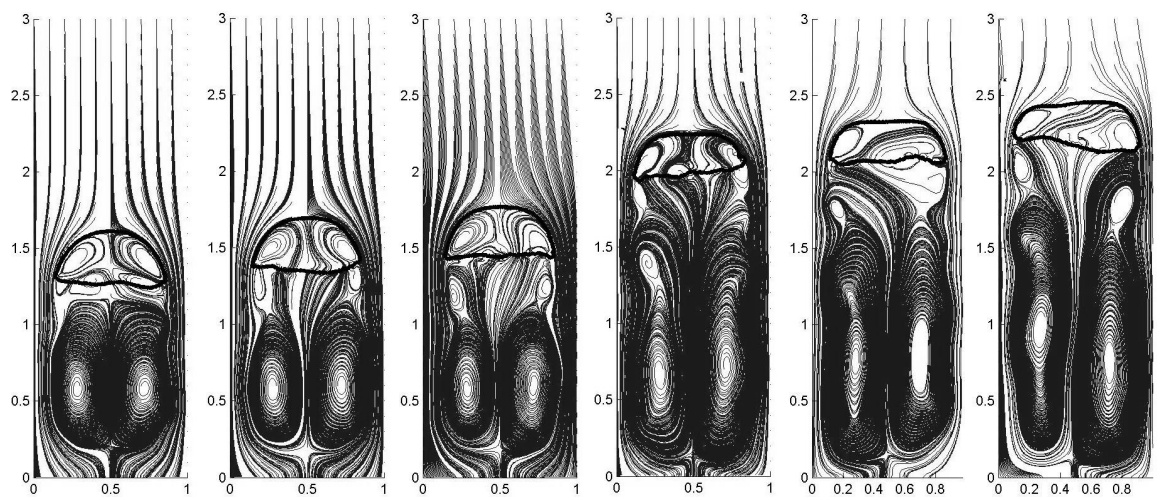

Figure 2. The rise of a wobbling bubble. Re $=1100$, Eo $=3.0 ; \rho_{1} / \rho_{2}=10^{3}, \mu_{1} / \mu_{2}=10^{2}$, $h=1 / 80$

into a single bubble. At this time, the interface conjunction forms a cusp singularity that is rapidly smoothed out by viscosity and surface tension.

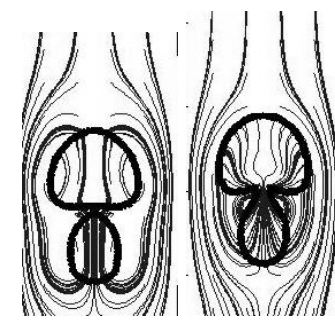

(a)

(b)

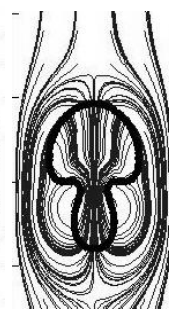

(c)

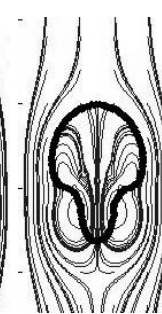

(d)

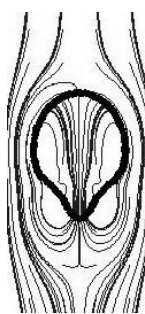

(e)

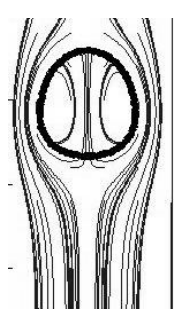

(f)

Figure 3. Merger of two spherical bubbles; $R e=2, E o=1.2, \rho_{1} / \rho_{2}=10^{2}, \mu_{1} / \mu_{2}=$ $10, h=1 / 40$.

Bubble coalescence in spherical shape regime is shown in Figure 3. Due to considerable rigidity of the bottom of the upper bubble, the liquid rather quickly becomes squeezed out of the space between the bubbles, and the bubbles merge.

In ellipsoidal shape regime, the bottom of the upper bubble deforms under the influence of the lower bubble, thus, making it possible to preserve a thin liquid film between the bubbles. The upper bubble develops a dimpled-ellipsoidal rather than an ellipsoidal shape. When the bottom of the upper bubble cannot deform any more, the liquid film between the bubbles starts getting thinner, and, finally, the lower bubble merges with the upper one.

The results agree with the computations by (Chang et al. 1996), by (Tornberg 2000), by (Unverdi \& Tryggvason 1992) and compare favorably with the numerical predictions by (Delnoij el al. 1998) who found a qualitative agreement with available experimental data. 


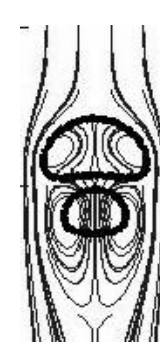

(a)

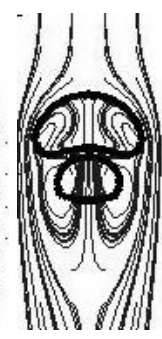

(b)

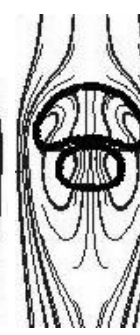

(c)

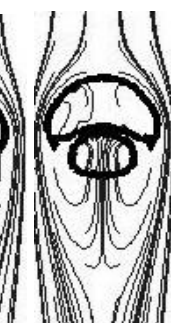

(d)

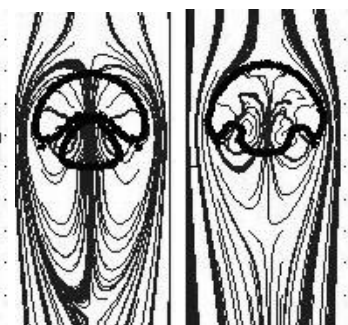

(e)

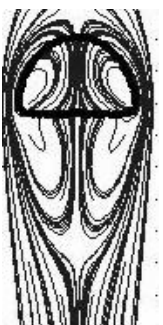

(f)

(g)

Figure 4. Merger of two ellipsoidal bubbles; $R e=20, E o=1.2, \rho_{1} / \rho_{2}=10^{2}$, $\mu_{1} / \mu_{2}=10, h=1 / 40$.

\section{Discussion}

We have presented the results of a computational study on two-dimensional bubble dynamics. Despite the seeming insufficiency of a two-dimensional model for the quantitative analysis of three-dimensional bubble evolution phenomena, we have been able to obtain a good qualitative agreement with the available experimental data. Since our numerical method captures the bubble interface as well as the surface tension effect and the mass conservation with the 2 nd order accuracy, we managed to recover all basic shape regimes within the experimentally predicted ranges of problem parameters. In particular, we successfully simulated the wobbling bubble regime remarkable by its unsymmetric vortex path pattern and a highly convective nature. Since the wobbling and the sphericalcap regimes are characterized by very high Reynolds numbers, it was essential to have a numerical method capable of dealing with convection-dominated flows. On the other hand, the method should be flexible enough to allow also a computation of a nearly Stokes flow (typical, e.g., for the case of a spherical bubble). We have demonstrated that such a flexibility can be maintained within the finite-element/level-set/operator-splitting framework.

In many cases, a good quantitative agreement has been observed (see (Smolianski et al. ) for a thorough comparison of our computational results with available experimental data). This, probably, means that a two-dimensional modeling of bubble dynamics is not so far from being realistic.

The preliminary study on the bubble coalescence phenomena also reveal that plausible results can be obtained already with two-dimensional simulations.

\section{Acknowledgments}

This work was supported by the grant 70139/98 of Tekes, the National Technology Agency of Finland.

\section{References}

Baker, G.R. and D.W. Moore, 1989, The rise and distortion of a two-dimensional gas bubble in an inviscid liquid. Phys. Fluids A 1, 1451-1459.

Batchelor, G.K., 1967, An Introduction to Fluid Dynamics. Cambridge University Press. 
Bhaga, D. and M.E. Weber, 1981, Bubbles in viscous liquids: shapes, wakes and velocities. J. Fluid Mech. 105, 61-85.

Chang, Y.C., T.Y. Hou, B. Merriman and S. Osher, 1996, A level set formulation of Eulerian interface capturing methods for incompressible fluid flows. J. Comput. Phys. $124,449-464$.

Clift, R.C., J.R. Grace and M.E. Weber, 1978, Bubbles, Drops and Particles. Academic Press.

Delnoij, E., J.A.M. Kuipers and W.P.M. van Swaaij, 1998, Computational fluid dynamics (CFD) applied to dispersed gas-liquid two-phase flows. In: Fourth European Computational Fluid Dynamics Conference ECCOMAS CFD'98, John Wiley \& Sons, Chichester, pp. 314-318.

Hartunian, R.A. and W. R. Sears, 1957, On the instability of small gas bubbles moving uniformly in various liquids. J. Fluid Mech. 3, 27-47.

Hnat, J.G. and J.D. Buckmaster, 1976, Spherical cap bubbles and skirt formation. Phys. Fluid 19, 182-194.

Moore, D.W., 1959, The rise of a gas bubble in a viscous liquid. J. Fluid Mech. 6, 113-130.

Sethian, A.J., 1999, Level Set Methods and Fast Marching Methods: Evolving Interfaces in Computational Geometry, Fluid Mechanics, Computer Vision, and Materials Science. Cambridge University Press.

Smolianski, A., 2001, Numerical Modeling of Two-Fluid Interfacial Flows, PhD thesis, University of Jyväskylä, ISBN 951-39-0929-8.

Smolianski, A., H. Haario, P. Luukka, Computational Study of Bubble Dynamics. To appear in the International Journal of Multiphase Flow.

Sussman, M., P. Smereka and S. Osher, 1994, A level set approach for computing solutions to incompressible two-phase flow. J. Comput. Phys. 114, 146-159.

Taylor, T.D. and A. Acrivos, 1964, On the deformation and drag of a falling viscous drop at low Reynolds number. J. Fluid Mech. 18, 466-476.

Tornberg, A.K., 2000, Interface Tracking Methods with Application to Multiphase Flows. $\mathrm{PhD}$ thesis, Royal Institute of Technology, Stockholm.

Unverdi, S.O. and G. Tryggvason, 1992, A front-tracking method for viscous, incompressible, multi-fluid flows. J. Comput. Phys. 100, 25-37.

Walters, J.K. and J.F. Davidson, 1962, The initial motion of a gas bubble formed in an inviscid liquid. Part 1. The two-dimensional bubble. J. Fluid Mech. 12, 408-417.

Walters, J.K. and J.F. Davidson, 1963, The initial motion of a gas bubble formed in an inviscid liquid. Part 2. The three-dimensional bubble and the toroidal bubble. J. Fluid Mech. 17, 321-336.

Wegener, P.P. and J.Y. Parlange, 1973, Spherical-cap bubbles. Ann. Rev. Fluid Mech. 5, 79-100. 